\title{
A weighted APIT localization algorithm based on vector similarity
}

\author{
Li He, Zhiwei Kang \\ College of Computer Science and Electronic Engineering \\ Hunan University \\ Changsha 410082, China \\ e-mail: jt_zwkang@hnu.edu.cn
}

\begin{abstract}
To reduce incorrect decisions of PIT test (PointIn-Triangulation Test) and localization error of APIT algorithm, a weighted APIT algorithm is proposed based on vector similarity. Firstly, for better distance precision, the estimated distances between nodes computed from RSSI attenuation model are corrected by the least square method. Secondly, a center of gravity method is proposed for PIT test, which decreases InToOut and OutToIn error. Finally, a weight is introduced based on vector similarity for the different reliability of triangular regions. The simulation result shows that the positioning error of ASG-APIT algorithm decreased by $43 \%, 17 \%$ and $13 \%$ respectively, compared with APIT, MIX-APIT and RAPIT algorithm.
\end{abstract}

Keywords-APIT algorithm, center of gravity method, weight based on vector similarity

\section{INTRODUCTION}

According to whether or not the distance between nodes is measured, localization algorithms are divided into the range-based and the range-free in wireless sensor network [1, 2]. The typical range-free algorithms contain DV-Hop [3], APIT [4, 5], and MDS-MAP [6], and centroiding algorithm [7] and so on. The low density of anchors has an adverse impact on localization coverage in APIT algorithm, but it is more likely to increase InToOut or OutToIn error under the high density of sensor nodes.

Aiming at the incorrect decisions of PIT test (Point-InTriangulation Test) and low positioning accuracy of APIT algorithm, some improved algorithms were presented. To increase localization coverage, Chen[8] proposed a mixed localization algorithm based on RSSI (Received Signal Strength Indicator) APIT. In order to reduce InToOut or OutToIn error, Zeng[9] proposed a new PIT test method based on cosine theorem. In [10, 11], a weighted APIT algorithm based on RSSI was presented in two algorithms respectively, and the unknown nodes that cannot be located will be confirmed by modified DV-Hop algorithm in [11]. However, the two algorithms both ignore the different credibility of the areas in which a node resides and fault tolerance of OutToIn error.

The paper proposes a weighted APIT localization based on vector similarity and the center of gravity method (VSGAPIT). The displacement relationship between unknown

\footnotetext{
* Corresponding author: Zhiwei Kang (jt_zwkang@hnu.edu.cn)
}

node and triangle vertices is used to determine whether the unknown node is in the triangle formed by connecting three neighboring anchors. The unknown nodes are not need to transmit its data to all neighboring nodes, which can save energy of nodes. A weight of vector similarity is introduced to improved localization accuracy.

\section{APIT LOCALIZATION ALGORITHM}

APIT (approximate point-in-triangulation test) algorithm is a method to narrow down the localization area in which a target node resides. In APIT algorithm, the first step is APIT test, it is a way to determine an unknown node whether is inside the triangle formed by three anchors. APIT test is repeated with different anchor combinations until all combinations are chosen. At this point, the centroid of overlap region of those triangles in which a node resides is chosen as the estimated position of unknown node. The critical step of the algorithm (APIT test) affects its localization precision.

However, the incorrect decisions of APIT test are often made in APIT algorithm for uneven distribution of nodes. It judges an interior node outside the triangle (InToOut) or judges an outside one inside the triangle (OutToIn), and OutToIn have greater probability under the uneven node density.

\section{ASG-APIT ALGORITHM}

\section{A. Center of gravity method judging inner point}

In APIT algorithm, unknown nodes need to receive RSSI data from all neighbor nodes for APIT test, which increases communication traffic of sensor network and InToOut or OutToIn errors. For avoiding the step, a center of gravity method is proposed to replace APIT test in ASG-APIT. In Fig. 1, the $\triangle A B C$ is formed by three anchors $(A, B$ and $C)$. We select $A$ as zero, and then the coordinates of any point $M$ is obtained by (1).

$$
M-A=p * \overrightarrow{(C-A)}+q * \overrightarrow{(B-A)}
$$

In (1), $p$ and $q$ are the offset factor of vectors. If $p$ or $q$ is negative, $M$ is outside the triangle; if $p+q=1, M$ lays on the 
edge $B C$ of triangle $\triangle A B C$. Therefore, we can get the criterion shown in (2) that $\mathrm{M}$ is inside the triangle.

$$
\left\{\begin{array}{l}
p>=0 \\
q>=0 \\
p+q<=1
\end{array}\right.
$$

We assume $\overrightarrow{v 0}=C-A, \overrightarrow{v 1}=B-A, \overrightarrow{v 2}=M-A$, then the dot product of (1) and $\overrightarrow{v 0}$ or $\overrightarrow{v 1}$ is

$$
\left\{\begin{array}{l}
\overrightarrow{v 2} \cdot \overrightarrow{v 0}=\mathrm{p} *(\overrightarrow{v 0} \cdot \overrightarrow{v 0})+q *(\overrightarrow{v 1} \cdot \overrightarrow{v 0}) \\
\overrightarrow{v 2} \cdot \overrightarrow{v 1}=\mathrm{p} *(\overrightarrow{v 0} \cdot \overrightarrow{v 1})+q *(\overrightarrow{v 1} \cdot \overrightarrow{v 1})
\end{array}\right.
$$

The solution of (3) is

$$
\left\{\begin{array}{l}
p=\frac{((\overrightarrow{v 1} \cdot \overrightarrow{v 1})(\overrightarrow{v 2} \cdot \overrightarrow{v 0})-(\overrightarrow{v 1} \cdot \overrightarrow{v 0})(\overrightarrow{v 2} \cdot \overrightarrow{v 1}))}{((\overrightarrow{v 0} \cdot \overrightarrow{v 0})(\overrightarrow{v 1} \cdot \overrightarrow{v 1})-(\overrightarrow{v 0} \cdot \overrightarrow{v 1})(\overrightarrow{v 1} \cdot \overrightarrow{v 0})} \\
q=\frac{((\overrightarrow{v 0} \cdot \overrightarrow{v 0})(\overrightarrow{v 2} \cdot \overrightarrow{v 1})-(\overrightarrow{v 0} \cdot \overrightarrow{v 1})(\overrightarrow{v 2} \cdot \overrightarrow{v 0}))}{((\overrightarrow{v 0} \cdot \overrightarrow{v 0})(\overrightarrow{v 1} \cdot \overrightarrow{v 1})-(\overrightarrow{v 0} \cdot \overrightarrow{v 1})(\overrightarrow{v 1} \cdot \overrightarrow{v 0}))}
\end{array}\right.
$$

where $\overrightarrow{v 2} \cdot \overrightarrow{v 0}=|A M| *|A C| * \cos a$, and $\alpha$ is got by cosine theorem. To improve distance precision, $|A C|$ and $|A B|$ are the Euclidean distances, and $|A M|,|B M|$ and $|C M|$ are the corrected estimated distances between nodes.

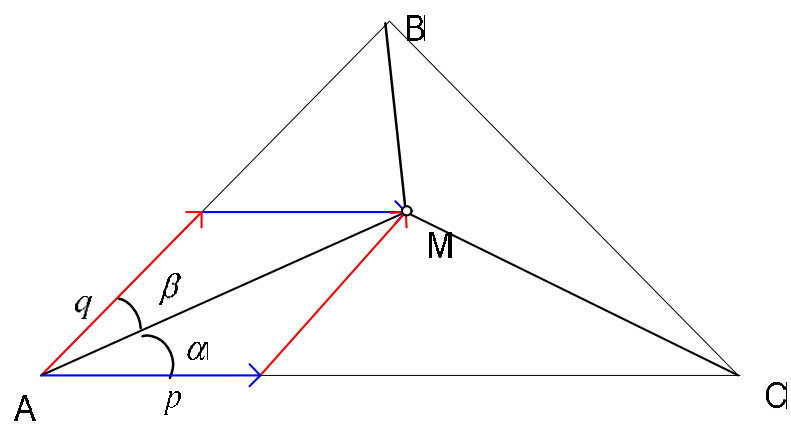

Fig.1. Center of gravity method

\section{B. Correct distance by least square method}

The high precision of the distances between nodes is important for the center of gravity method. However, the distances between nodes computed by the RSSI attenuation model is not sufficient, because RSSI changes randomly in outdoor. To improve the accuracy of estimated distance, the error compensation of estimated distance is achieved by the least square method.

$$
y=a x+b
$$

The estimated distances between five random anchor and its neighbor anchors are selected for computing the parameters of linear fitting (5). Assuming the number of neighbor anchors of node $\mathrm{i}=(1,2 \ldots 5)$ is $n_{i}$, we can get the Euclidean distances and estimated distances between the selected nodes $\mathrm{i}$ and its neighbor anchors, they are $e_{i j}$ and $x_{i j} \quad \mathrm{i}=(1,2, \ldots, 5), \mathrm{j}=\left(1,2, \ldots, n_{i}\right)$. The gap between $e_{i j}$ and $x_{i j}$ is $y_{i j}$. According to the least square method, the parameters of linear fitting equation (5) are computed by (6).

$$
\left\{\begin{array}{c}
\hat{a}=\frac{\left(\sum_{i=1}^{5} \sum_{j=1}^{n_{i}} x_{i j}{ }^{2}\right)\left(\sum_{i=1}^{5} \sum_{j=1}^{n_{i}} y_{i j}\right)-\left(\sum_{i=1}^{5} \sum_{j=1}^{n_{i}} x_{i j}\right)\left(\sum_{i=1}^{5} \sum_{j=1}^{n_{i}} x_{i j} y_{i j}\right)}{\left(\sum_{i=1}^{5} n_{i}\right)\left(\sum_{i=1}^{5} \sum_{j=1}^{n_{i}} x_{i j}{ }^{2}\right)-\left(\sum_{i=1}^{5} \sum_{j=1}^{n_{i}} x_{i j}\right)^{2}} \\
\hat{b}=\frac{\left(\sum_{i=1}^{5} n_{i}\right)\left(\sum_{i=1}^{5} \sum_{j=1}^{n_{i}} x_{i j} y_{i j}\right)-\left(\sum_{i=1}^{5} \sum_{j=1}^{n_{i}} x_{i j}\right)\left(\sum_{i=1}^{5} \sum_{j=1}^{n_{i}} y_{i j}\right)}{\left(\sum_{i=1}^{5} n_{i}\right)\left(\sum_{i=1}^{5} \sum_{j=1}^{n_{i}} x_{i j}{ }^{2}\right)-\left(\sum_{i=1}^{5} \sum_{j=1}^{n_{i}} x_{i j}\right)^{2}}
\end{array}\right.
$$

The corrected way is shown as (7) in which $d$ is the estimated distance between nodes, and $\Delta_{i}$ is calculated by (5).

$$
\widehat{d}= \begin{cases}d+\left|\Delta_{i}\right| & \Delta_{i<0} \\ d-\Delta_{\mathrm{i}} & \Delta_{i}>0\end{cases}
$$

\section{Weighted centroid algorithm based on vector similarity}

Taking into account the different reliability of triangles formed by anchors, a weight of vector similarity is introduced. In Fig. 2, $A, B$ and $C$ are anchors. The center of gravity (point $M$ ) of $\triangle A B C$ is chosen as the estimated position of the unknown node $p_{i} i=(1,2,3)$, so the closeness between $M$ and $p_{i}$ is positively related to the localization accuracy of $p_{i}$.

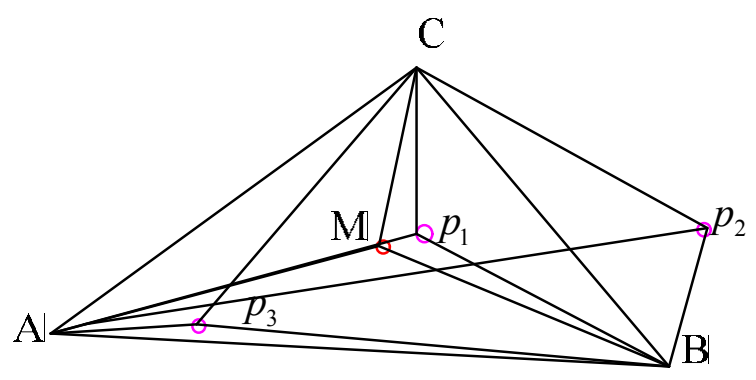

Fig.2. Weight of vector similarity 


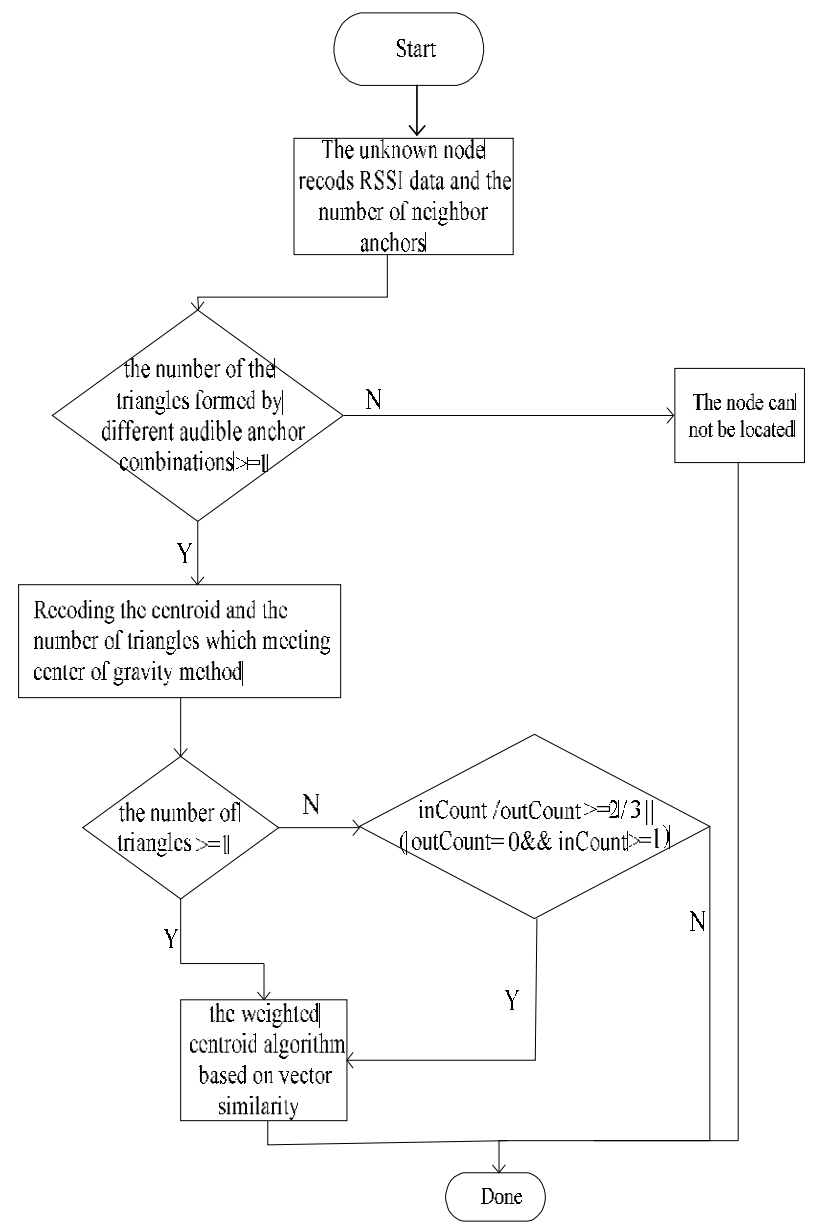

Fig.3. ASG-APIT algorithm

The coordinates of $A, B$ and $C$ respectively are $\left(x_{\mathrm{a}}, y_{\mathrm{a}}\right),\left(x_{\mathrm{b}}\right.$, $\left.y_{\mathrm{b}}\right)$ and $\left(x_{\mathrm{c}}, y_{\mathrm{c}}\right)$, and $M$ is $\left(x_{\mathrm{m}}, y_{\mathrm{m}}\right)$. We get two vectors, they are $\vec{\varepsilon}\left(d_{a}, d_{b}, d_{c}\right)$ and $\vec{\gamma}\left(d^{\prime}{ }_{i a}, d^{\prime}{ }_{i b}, d^{\prime}{ }_{i c}\right) i=(1,2,3)$. The members of the two vectors separately are the Euclidean distances computed by (8) between $M$ and triangle vertices and the correctional estimated distances between $p_{i}$ and triangle vertices.

$$
\left\{\begin{array}{l}
d_{a}=\sqrt{\left(x_{m}-x_{a}\right)^{2}+\left(y_{m}-y_{a}\right)^{2}} \\
d_{b}=\sqrt{\left(x_{m}-x_{b}\right)^{2}+\left(y_{m}-y_{b}\right)^{2}} \\
d_{c}=\sqrt{\left(x_{m}-x_{c}\right)^{2}+\left(y_{m}-y_{c}\right)^{2}}
\end{array}\right.
$$

We use $\delta$ computed by (9) to show the similarity between $\vec{\gamma}$ and $\vec{\varepsilon}$, then $1 / \delta^{2}$ is selected as the weight of positioning for enhancing the fault tolerance of OutToIn error

$$
\delta_{i}=\sqrt{\left(d_{a}-d_{i a}^{\prime}\right)^{2}+\left(d_{b}-d_{i b}^{\prime}\right)^{2}+\left(d_{c}-d_{i c}^{\prime}\right)^{2} / 3}
$$

In ASG-APIT algorithm, the center of gravity of the area formed by three intersections is involved in equation (10), if we can get three intersections of three circles whose centers are three anchors and radii are corrected distances between unknown node and anchors. We assume that the centroids of triangles selected by center of gravity method are $\left(x_{1}, y_{1}\right),\left(x_{2}\right.$, $\left.y_{2}\right)$... and $\left(x_{\mathrm{n}}, y_{\mathrm{n}}\right)$, so the estimated position of the unknown node is got by (10).

$$
\begin{gathered}
(x, y)=\left(\sum_{i=1}^{n}\left(1 / \delta_{i}^{2} * x_{i}\right) / \sum_{i=1}^{n} 1 / \delta_{i}^{2},\right. \\
\left.\sum_{i=1}^{n}\left(1 / \delta_{i}^{2} * y_{i}\right) / \sum_{i=1}^{n} 1 / \delta_{i}^{2}\right)
\end{gathered}
$$

In conclusion, the steps of ASG-APIT algorithm are shown in Fig. 3. The inCount is the number of neighbor nodes meeting the APIT test, and outCount is the opposite number.

\section{SIMULATION RESULTS AND ANALYSIS}

\section{A. Simulation environment}

In order to evaluate the algorithm performance, the MATLAB 2009a platform is used to simulate APIT, RAPIT [10], MIX-APIT[11], and ASG-APIT algorithm. The simulation scenario is a $1000 * 1000 \mathrm{~m}$ square region with 300 nodes deployed. To ensure that the experiment is as true to reality as possible, the Logarithmic Attenuation Model[12] is utilized. Every experiment under different conditions is simulated 10 times to get the mean.

The localization error between estimated positions $\left(x_{i}^{\prime}, y_{i}^{\prime}\right)$ of nodes and actual positions $\left(x_{\mathrm{i}}, y_{\mathrm{i}}\right)$ is assessed by (11) in which $R$ is the communication radius.

$$
\text { error }=\frac{1}{N R} \sum_{i=1}^{N} \sqrt{\left(x_{i}^{\prime}-x_{i}\right)^{2}-\left(y_{i}^{\prime}-y_{i}\right)^{2}}
$$

\section{B. Results and Analysis}

In homogeneous sensor network, the communication radius is $200 \mathrm{~m}$ between normal nodes and anchors, and the number of anchors increases from 40 to 70 . Fig. 4 is shown that the average localization error of overall algorithms decrease with increasing of the number of anchors. The localization accuracy of ASG-APIT algorithm improves significantly, its positioning error reduces by $12.3 \%, 7.25 \%$ and $4.6 \%$, compared with APIT, MIX-APIT and RAPIT.

In heterogeneous network, the communication range of anchors is the double of unknowns, and there are 60 anchors among 300 nodes. The Fig. 5 shows that the positioning error of APIT algorithm increases gradually with the increasing of communication range, but ASG-APIT reduces slowly. Compared with APIT, MIX-APIT and RAPIT, the positioning error of ASG-APIT reduces by $43 \%, 17 \%$ and $13 \%$ respectively. 


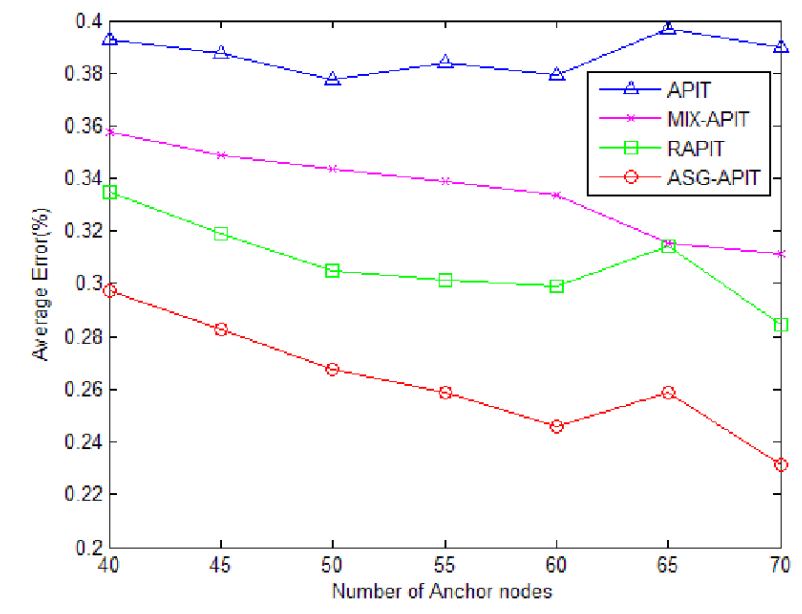

Fig.3. Average localization error

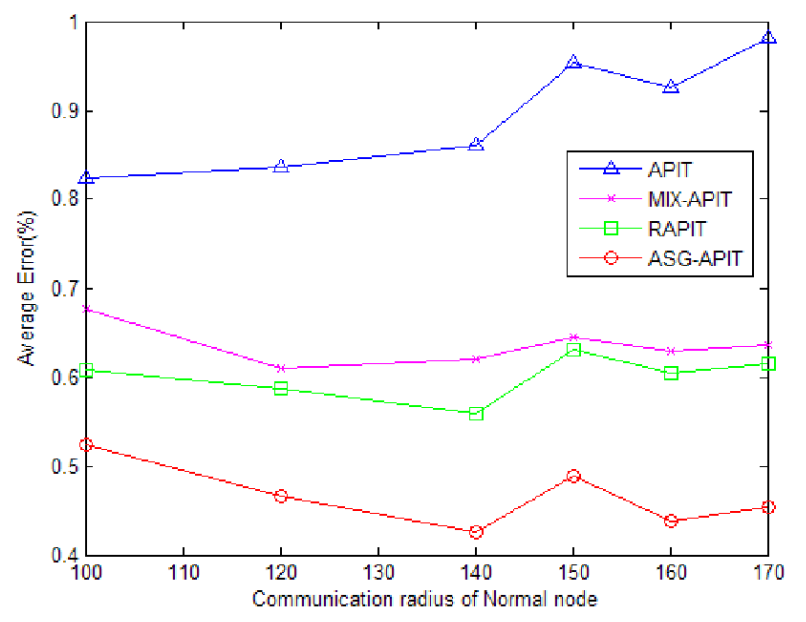

Fig.4. Average localization error

\section{CONCLUSION}

This paper proposes a weighted APIT algorithm based on vector similarity from the study on PIT test and the method for computing the coordinates of unknown. The algorithm builds the judging criteria of PIT test by using the relative positions between unknown node and vertexes of triangle, which saves energy of nodes and decreases the incorrect decisions of PIT test. The corrected model of estimated distance, which is established on the basis of $n$ group of measured data between anchors, decreases the ranging error between nodes. The weighted centroiding algorithm based on vector similarity is used to get the coordinates of unknown node, which enhances the resistance to OutToIn error and positioning precision of the algorithm. The results show that the positioning accuracy of ASG-APIT algorithm increases dramatically under same environment, compared with APIT, RAPIT and MIX - APIT algorithm.

\section{ACKNOWLEDGMENT}

This work is supported by Science and Technology Planning Project of Hunan Province of China (2014WK2002).

\section{REFERENCES}

[1] Y. Peng, D. Wang, "A review: wireless sensor networks localization," Journal of Electronic measurement and Instrument, Vol. 25, pp. 389396, 2011.

[2] F L. Lewis, "Smart Environments: Technologies, Protocols, and Applications," John Wiley, New York , pp. 11-46, 2014.

[3] D. Niculescu, B. Nath, "DV based positioning in ad hoc networks," Telecommunication Systems, Vol. 22, pp. 267-280, 2003.

[4] T. He, C. Huang, B. M. Blum, J. A. Stankovic, and T. Abdelzaher, "Range-free localization schemes for large scale sensor networks," 9th annual international conference on Mobile computing and networking, pp.81-95, September 2003.

[5] J. Wang, J. Q. Fu. "Study on localization algorithm for wireless mobile node based on APIT and particle filter," Transducer and Microsystem Technologies, Vol. 9, pp. 024, 2011.

[6] N. Bulusu, J. Heidemann, and D. Estrin, "GPS-less low-cost outdoor localization for very small devices, " IEEE. J. Personal Communications, Vol. 7, pp. 28-34, 2000.

[7] Y. Shang, W. Ruml, Y. Zhang, and M. P. Fromherz, "Localization from mere connectivity, " 4th ACM international symposium on Mobile ad hoc networking \& computing. ACM, 2003.

[8] B. Chen, J. Sun, W. B. Xu, and J. Xu, "A Mixed Localization Algorithm Based on RSSI and APIT with Fitness Analysis and Optimization," IEEE International Symposium on Distributed Computing and Applications to Business, Engineering \& Science (DCABES), 2012.

[9] F. Zeng, M. Yu, C. Zou, J. Gong, "An Improved Point-inTriangulation Localization Algorithm Based on Cosine Theorem," 8th International Conference on Wireless Communications, Networking and Mobile Computing (WiCOM), pp.1-4, 2012.

[10] X. F. Cheng, L. Zhong, "A new node self-localization algorithm based RSSI for wireless sensor networks," 5th International Conference on Computational and Information Sciences (ICCIS), IEEE, pp. 1616-1619. 2013.

[11] W. Liang, X. W. Jian, "A mixed wireless sensor networks localization algorithm," Transducer and Microsystem Technologies,Vol. 32, pp.102-105, 2013.

[12] Q. Chen, "Analysis of the Localization Performance in Different Propagation Models about APIT Algorithm," Dianzi Keji-Electronic Science and Technology, Vol.26, pp. 86-89 2013. 